\title{
Graphical Analysis of Electromagnetic Coupling on B-737 and B-757 Aircraft for VOR and LOC IPL Data
}

\author{
Madiha Jafri ${ }^{1}$, Jay Ely ${ }^{2}$, and Linda Vahala ${ }^{1}$ \\ ${ }^{1}$ Department of Electrical \& Computer Engineering, Old Dominion University, Norfolk, VA 23529 \\ ${ }^{2}$ NASA Langley Research Center, Hampton, VA 23665
}

\section{Introduction:}

The use of portable wireless technology has increased dramatically over the past few years. PEDs may act as transmitters and their signals may be detected by the various radio receiver antennas installed on the aircraft. Measurement of the radiated field coupling between passenger cabin locations and aircraft communication and navigation receivers, via their antennas is defined herein as interference path loss (IPL) ${ }^{1}$. Personnel from NASA Langley Research Center and Delta Air Lines recently completed extensive IPL measurements on several Boeing 757 airplanes. Prior research includes analysis of measurements on United Airlines Boeing 737 airplanes as well.

Compared to the B-737, the B-757 is composed of a longer fuselage, more exit doors, windows, emergency exits and the aircraft antenna placements are different. Figure 1 shows the interior schematics of both B-737 and B-757 where the nose is on the left of both schematics. The objective of this paper is to analyze the IPL data measured, to better understand the impact on coupling levels based on the different locations of the aircraft radio antennas on the B-757 and B-737 airplanes, and to provide a basis for future fuzzy logic modeling of airplane IPL. This effort will build upon previous fuzzy modeling of IPL data for B-737 airplane data. ${ }^{2}$ Systems studied and compared include the Instrument Landing System Localizer (LOC) and VHF Omniranging (VOR) system.

\footnotetext{
1 Jafri, Madiha, J. Ely, L. Vahala. "Graphical and Statistical Analysis of Airplane Passenger Cabin RF Coupling Paths to Avionics." 22nd Digital Avionics Systems Conference, Indianapolis, IN, October 12 - 16, 2003.

2 Jafri, Madiha, J. Ely, L. Vahala, "Fuzzification of Electromagnetic Interference Patterns Onboard Commercial Airliners Due to Wireless Technology," 2004 IEEE International Symposium on Antennas and Propagation and USNC/URSI National Radio Science Meeting, Monterey, California, June 20-26, 2004.
} 


\section{Discussion:}

Figure 2 shows a comparison of IPL data for B-757 VOR and B-737 VOR/LOC. In both cases, the aircraft antennas are located on the tip of the aircraft tail and are horizontally polarized. In the figures, the $\mathrm{x}$-axis represents the window locations from window $\# 1$ to the end of the airplane (\#33 in B-737, \#53 in B-757); while the y-axis represents the test locations within the rows, where "W-h" represents window location in horizontal polarization to "I-h" represents aisle, also in horizontal polarization. The y-axis also includes window through aisle data in vertical polarization as well. The z-axis, represented by the color bar on the right of the plots, represents pathloss in $\mathrm{dB}$, where the red regions represent areas of greatest coupling (lowest pathloss), while the blue regions represents lesser coupling.

In the case of B-757 VOR, the regions of greatest coupling occur in the row of windows $\# 20$ to \#25. Two emergency exits are located at \#23 and \#24, which are the source of leakage for this particular system. Figure 2 (right) shows the results previously obtained for VOR/LOC system antenna on B-737. These results are interesting because the greatest levels of coupling are just forward of the over-wing exits on both airplanes. In both cases, highest coupling would have been hypothesized to be at the rear exit of the aircraft, closest to the VOR antenna location. On the B-737, the greatest levels of coupling are present alongside the windows located in the front of the aircraft. Therefore, it is seen that although the antenna locations of B-757 VOR and B-737 VOR/LOC are similar, the interference patterns for the two airplanes differ in their dependence upon polarization. It's also important to note that maximum coupling occurs inside the cabin, away from the windows. (However, this data does not include window and door IPL sweeps.)

The second objective was to compare the effects of mounting an antenna for a given frequency band at two different aircraft locations. The LOC system shares the VOR antenna located on the tip of the tail on B-737, as noted previously; however, the LOC antenna is located separately inside the nose radome of the B-757. Figure 3 (left) shows the IPL data obtained on B757 in both horizontal (top) and vertical (bottom) test antenna polarizations for both LOC (red 
circle, located in nose) and VOR (blue star, located on tail) systems on the same aircraft. Although the antennas are located on the opposite sides of the aircraft (nose vs. tail), the IPL measurements along the windows are amazingly close to each other in both polarizations. The greatest coupling values for both systems occur near the L1 and L3 doorways as well as near the emergency exits (\#23,\#24). A slight peak in coupling also occurs near the L2 doorway (\#14).

For the B-737, in which the VOR/LOC system is located on the tip of the tail, the IPL data from horizontal polarization does not deviate much from $-65 \mathrm{~dB}$; however, much deviation can be observed in vertical polarization (Figure 3, right). Comparison of the LOC (red lines in left column) vs. the LOC in B-737 shows that when the location of the same system is changed (LOC in nose of B-757 while in tail of B-737), the overall IPL pattern changes across the windows of the aircraft in horizontal polarization; however, the results from vertical polarization produce the same locations for the peak values of coupling: near the doors and emergency exit(s).

\section{Conclusion and Future Work:}

The results from this paper will be incorporated in fuzzy modeling of EMI patterns on structurally different aircraft. Fuzzy logic methods may provide a means to assess IPL characteristics of aircraft that have not been subject to expensive measurement or modeling processes and may also be useful for estimating the merit of aircraft design changes intended to minimize the potential for EMI. The author would like to thank Delta Airlines for their support in the test taking process and analysis.

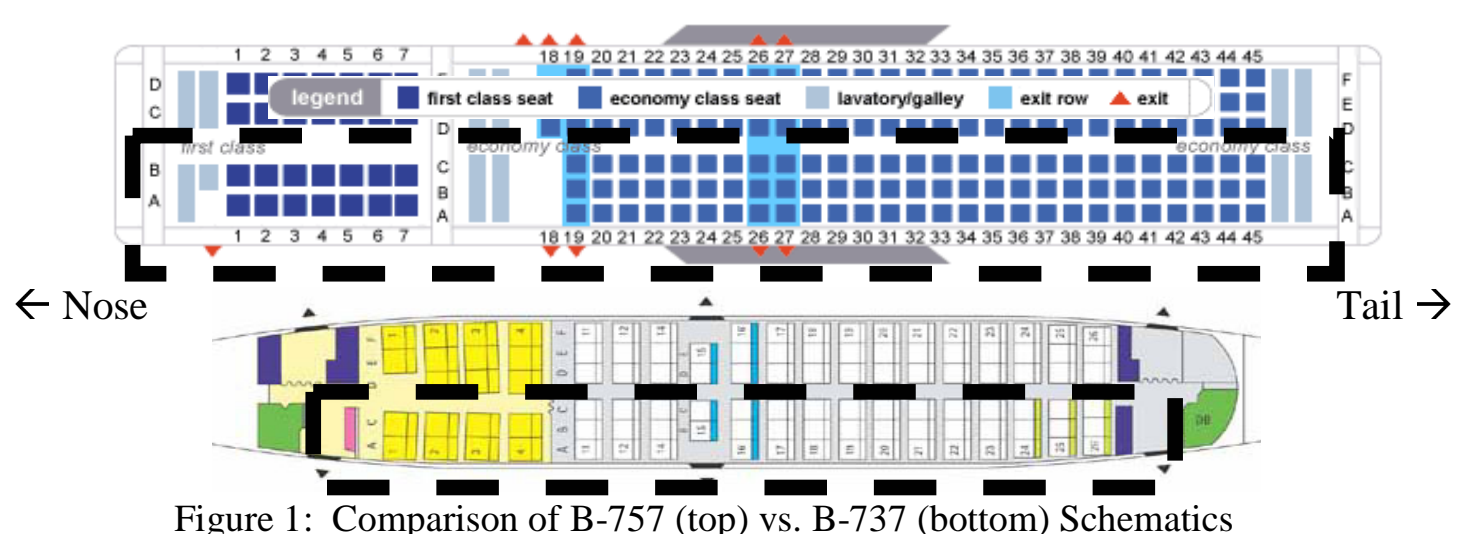

Figure 1: Comparison of B-757 (top) vs. B-737 (bottom) Schematics 


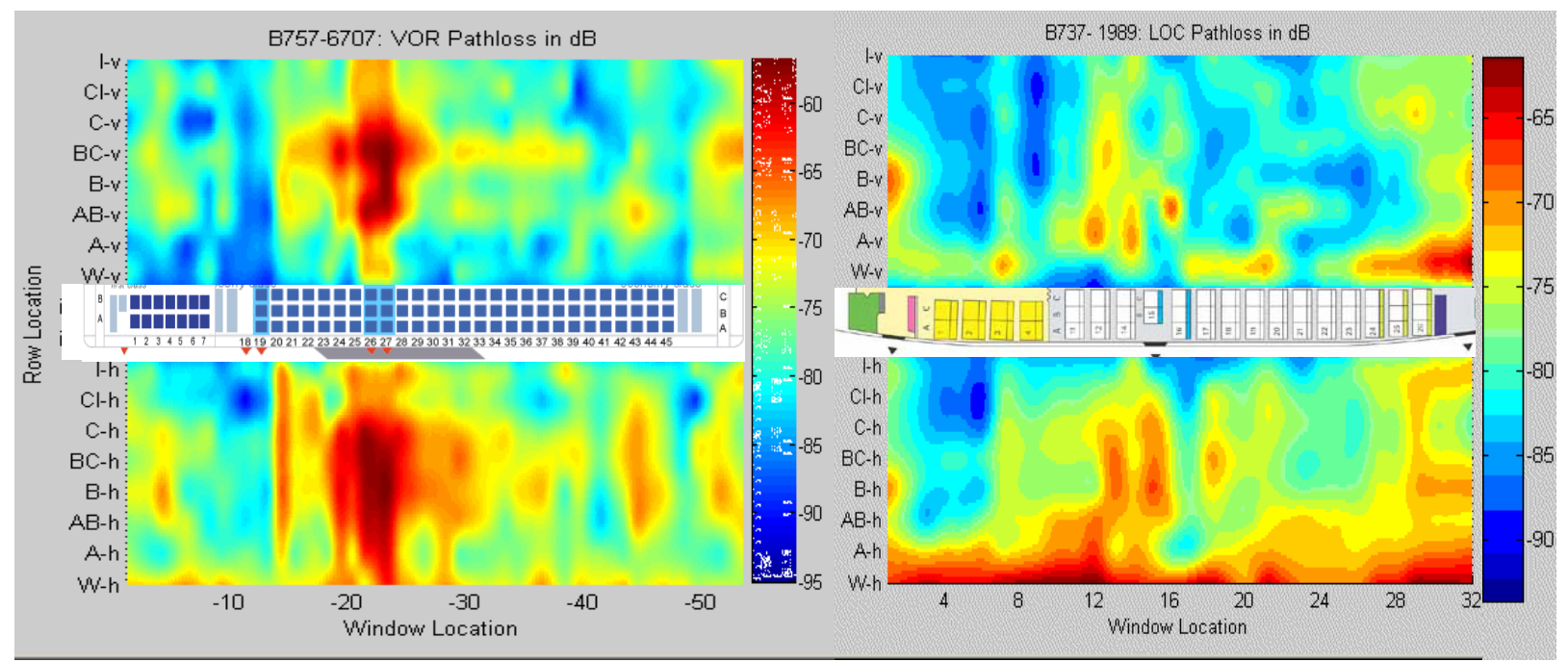

Figure 2: Comparison of B-757:VOR (left) and B-737:VOR/LOC (right) where both antenna systems are located on the tip of the tail of aircraft
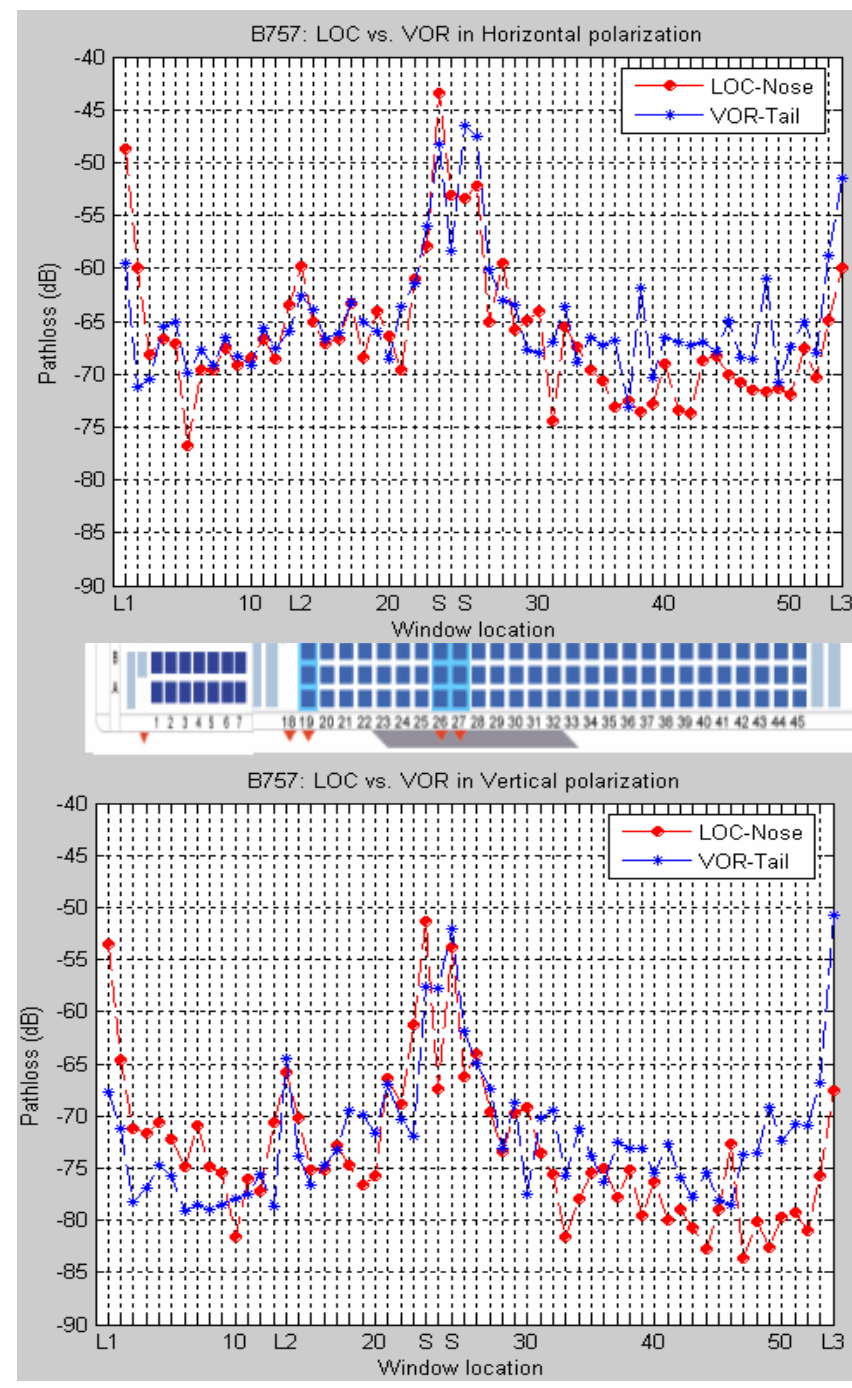

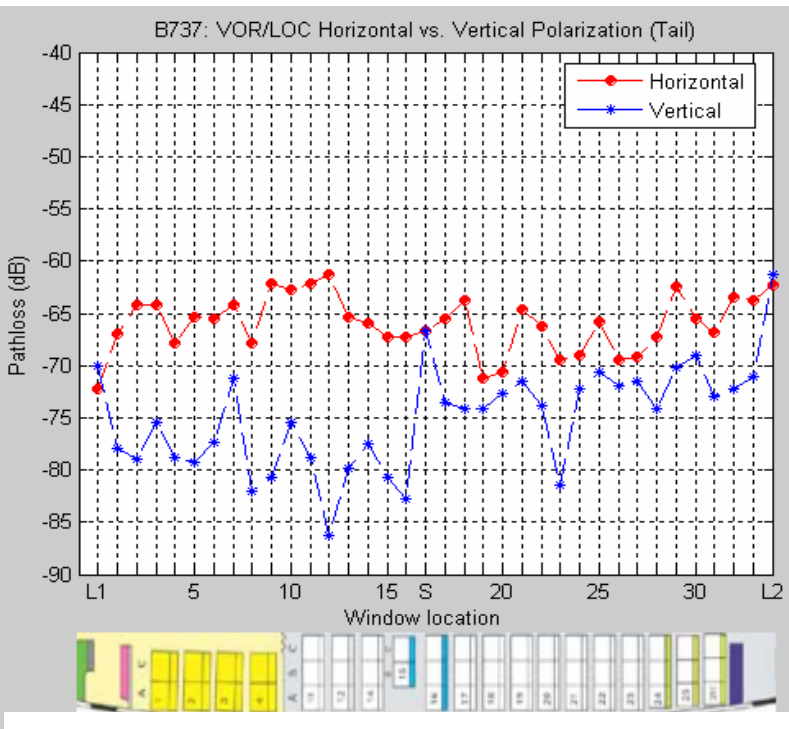

Figure 3:

(Left: top \& bottom) Comparison of LOC vs.

VOR on B-757 in horizontal and vertical polarizations (respectively). LOC is located in the nose while VOR is located on the tip of the tail of aircraft $(\mathrm{S}=$ exit sweeps)

(Right: top) Results for LOC in B-737 in horizontal and vertical polarizations. LOC is located on the tip of the tail on B-737. Used to compare with the red-circled lines in the left plots. 